\title{
Corrosão por ação de cloretos em estruturas de concreto armado: uma análise das publicações da última década
}

\begin{tabular}{c}
\hline TÁSSIA FANTON - DoutORANDA EM ENGENHARIA CIVIL \\
ORCID: https://orcid.org/0000-0002-3938-4529 \\
\hline ROGÉRIO CATTELAN ANTOCHEVES DE LIMA - ProfESSOR DoutOR \\
ORCID: https://orcid.org/0000-0001-6622-2210
\end{tabular}

Programa de Pós-Graduaç̃o em Engenharia Civil Universidade Federal de Santa Maria (PPGEC UFSM)

\author{
FRANCIELE MÜLLER RIBEIR0 - Mestranda em EngenhariA OceânICA \\ ORCID: HTTPS://ORCID.ORG/0000-0001-6480-5738 \\ Programa de Pós-Graduação em Engenharia Oceânica \\ Universidade Federal do Rio Grande (PPGEO FURG)
}

\begin{abstract}
RESUMO
A PRESENTE PESQUISA DESENVOLVEU UM ESTUDO DA BIBLIOGRAFIA DISPONIVEL SOBRE A TEMÁTICA DE CORROSÃO DE ARMADURAS EM ESTRUTURAS DE CONCRETO ARMADO POR AÇÃO DE ÍONS CLORO. OS DADOS FORAM COLETADOS NA Web of ScIENCE (WOS) No PERÍOdO dE 2009 a 2018, SENDO ENCONTRADAS 1100 PUBLICAÇÕES. FORAM IDENTIFICADAS AS ÁREAS QUE SÃO DE RELEVÂNCIA DENTRO DESSE TÓPICO, BEM COMO FOI REALIZADA UMA ANÁLISE DAS PUBLICAÇÕES MAIS CITADAS. AlÉM DISSO, AS PUBLICAÇÕES FORAM

ANALISADAS QUANTO À POSSIBILIDADE DE SEREM UM "HOT TOPIC". CONSTATOU-SE QUE OS MAIORES NÚMEROS DE PUBLICAÇÕES ESTÃO NA CHINA E Estados Unidos. A temÁtICA FOI CONSIDERADA UM "HOT TOPIC" NAS ÁREAS DE ENGENHARIA, CIÊNCIA DOS MATERIAIS, TECNOLOGIA DA CONSTRUÇÃO E ENGENHARIA METALÚRGICA. NO QUE SE REFERE AO CONTEÚDO DAS PUBLICAÇÕES MAIS CITADAS, DESTACA-SE O ESTUDO DE INIBIDORES DE CORROSÃO E MODELOS PROBABILÍSTICOS PARA A PREVISÃO DE VIDA ÚTIL.
\end{abstract}

Palavras-chave: bibliometria, corrosão de armaduras, cloretos, estruturas de concreto.

\section{INTRODUÇÃO}

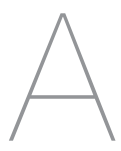

durabilidade das estruturas de concreto armado já

foi considerada ilimitada, uma vez que a armadura estaria protegida dos agentes agressivos. No entanto, por volta da década de 1970, esse entendimento mudou, haja vista que a indústria da construção civil começou a confrontar-se com uma gama de manifestações patológicas, sendo necessários elevados custos para o seu reparo. Esse cenário despertou o interesse do meio científico, de modo que a durabilidade das estruturas passou a ser estudada ainda na fase de projeto. (NEPOMUCENO, 2005).
Segundo Ribeiro e Cascudo (2018), a durabilidade de uma estrutura é função de determinados parâmetros, como a agressividade ambiental, as características da construção, os critérios de desempenho desejados e o tempo, ou seja, a vida útil requerida para uma estrutura. É válido destacar que a corrosão das armaduras é uma das manifestações patológicas mais frequentes nas estruturas de concreto armado e que sua evolução tende a comprometer significativamente a segurança estrutural de uma determinada obra.

Quando um concreto se encontra exposto em um ambiente rico em sais, ele pode ser contaminado através de agentes presentes no meio em que esse material foi inserido. Isso ocorre devido ao seu caráter poroso e a existência de uma solução alcalina na sua rede de poros; assim, os cloretos podem penetrar no interior do concreto, sendo transportados desde superfícies mais expostas até as camadas mais internas.

Tendo isso em mente, o presente trabalho visa realizar um estudo bibliométrico quantitativo das publicações referentes ao fenômeno de corrosão das armaduras em estruturas de concreto pela ação dos cloretos. Como contribuição científica, espera-se oferecer subsídio e informações para profissionais e pesquisadores da área, 
especialmente no sentido de alertar sobre a importância da temática na garantia de uma maior durabilidade e desempenho das estruturas, possibilitando, assim, construções com maior vida útil e sustentabilidade.

\section{DESENVOLVIMENTO}

A presente investigação foi desenvolvida a partir de uma pesquisa bibliométrica de cunho quantitativo, tendo como objetivo identificar as características das publicações científicas sobre a temática da corrosão de armaduras em estruturas de concreto armado por ação de cloretos. É válido destacar, que, de acordo com Araújo (2006), a bibliometria, técnica aplicada neste estudo, possui o intuito de quantificar, identificar, analisar e descrever uma série de padrões na produção de conhecimento científico sobre um tema específico. Logo, a bibliometria analisa a atividade científica por meio de um estudo quantitativo de publicações, auxiliando no conhecimento do estágio de uma área específica de pesquisa.

Os dados utilizados nesta pesquisa foram coletados por meio da plataforma WOS (Web of Science), utilizando o seguinte operador booleano (("reinforced concrete") AND corrosion AND structur $^{*}$ AND chlorid*). A busca foi delimitada para o período de 2009 a 2018 (10 anos).

\section{I Modelo conceitual}

As variáveis analisadas na presente pesquisa são: áreas temáticas, tipos de documentos, ano das publicações, autores, título das fontes, instituições, países, índice hb e índice m.

O banco de dados coletado na Web of Science (WOS) foi analisado uti- lizando-se os índices hb e m, os quais serviram de base para realizar uma análise dos "Hot Topics". O índice hb foi proposto por Hirsch (2005), o qual apresenta uma maneira de caracterizar a produção científica de um pesquisador. O índice hb é obtido por meio do número de citações de um tópico ou combinação em certo período, listados em ordem decrescente de citações, definindo-se o número de artigos com citações maiores ou iguais a esse número. Posteriormente, Banks (2006) propôs uma extensão do índice hb, o índice $\mathrm{m}$, obtido pela relação entre o índice hb e o período de anos em que se deseja obter as informações. Assim sendo, a partir do índice $\mathrm{m}$ têm-se as seguintes definições para a classificação dos "Hot Topics", (Tabela 1).

Conforme as definições de Banks (2006), foram considerados "Hot Topics" as combinações com índice $m \geq 2$.

$>$ Tabela 1 - Definições para a classificação de "Hot Topics"

\begin{tabular}{|c|c|}
\hline Índice m & Tópico/combinação \\
\hline $0,0<m \leq 0,5$ & $\begin{array}{l}\text { Pode ser de interesse } \\
\text { para pesquisadores de } \\
\text { um campo específico de } \\
\text { pesquisa, o qual engloba } \\
\text { uma comunidade pequena }\end{array}$ \\
\hline $0,5<m \leq 2,0$ & $\begin{array}{c}\text { Provavelmente pode se } \\
\text { tornar um "Hot Topic" como } \\
\text { área de pesquisa, no qual a } \\
\text { comunidade é muito grande } \\
\text { ou o tópico/combinação } \\
\text { apresenta características } \\
\text { muito interessantes }\end{array}$ \\
\hline$m \geq 2,0$ & $\begin{array}{l}\text { É considerado um "Hot } \\
\text { Topic", tópico exclusivo } \\
\text { com alcance não apenas } \\
\text { na sua própria área de } \\
\text { pesquisa, e é provável que } \\
\text { tenha efeitos de aplicação } \\
\text { ou características únicas }\end{array}$ \\
\hline Fonte: Banks (2006) & \\
\hline
\end{tabular}

\subsection{Etapas para coleta de dados}

A pesquisa foi dividida em sete etapas. Primeiramente, utilizou-se do operador booleano (("reinforced concrete") AND corrosion AND structur ${ }^{*}$ AND chlorid $\left.^{\star}\right)$, sendo delimitado o período de 2009 a 2018. Em seguida, o banco de dados da WOS foi utilizado para pesquisar as variáveis a serem analisadas.

Após essas ações, foram identificados os tópicos a serem combinados, por meio de uma breve análise das publicações. Foram, então, listados 10 tópicos aliados à análise da corrosão das armaduras de estruturas em concreto armado por ação de cloretos. Na quinta fase, a busca no sistema foi combinada para cada um dos temas relacionados à patologia da construção em questão para o período de 10 anos. Então, na sexta etapa, os índices hb e m foram calculados, classificando-se os "Hot Topics". Por fim, na última fase, realizou-se uma análise das 10 publicações mais citadas no período analisado.

\section{DISCUSSÃO E ANÁLISE DOS RESULTADOS}

Os resultados da pesquisa destacam as principais características da produção científica relacionada ao ataque de cloretos em estruturas de concreto armado. A pesquisa utilizando-se da base de dados e do operador, anteriormente apresentados, resultou em 1100 publicações.

\section{I Características gerais das publicações na Web of Science - WOS}

As características gerais das publicações relacionadas ao assunto são apresentadas de acordo com: áreas 


\begin{tabular}{|c|c|}
\hline \multicolumn{2}{|c|}{$\begin{array}{l}\text { Tabela } 2 \text { - Principais áreas } \\
\text { temáticas }\end{array}$} \\
\hline Áreas de pesquisa & Registros \\
\hline Engenharia Civil & 572 \\
\hline $\begin{array}{l}\text { Ciência dos Materiais } \\
\text { Multidisciplinar }\end{array}$ & 478 \\
\hline Tecnologia da Construção & 455 \\
\hline $\begin{array}{c}\text { Metalurgia e Engenharia } \\
\text { Metalúrgica }\end{array}$ & 106 \\
\hline Engenharia Mecânica & 95 \\
\hline $\begin{array}{l}\text { Testes de Caracterização } \\
\text { Ciência dos Materiais }\end{array}$ & 57 \\
\hline Eletroquímica & 54 \\
\hline $\begin{array}{l}\text { Ciência dos Materiais } \\
\text { Compósitos }\end{array}$ & 54 \\
\hline Engenharia Multidisciplinar & 45 \\
\hline Mecânica & 23 \\
\hline Fonte: Web of Science (2019) & \\
\hline
\end{tabular}

temáticas, tipos de documentos, ano de publicação, autores, título das fontes de publicação, instituições e países.

\section{I.I ÁREAS TEMÁTICAS DE PUBLICAÇÃo E TIPOS DE DOCUMENTOS}

A Tabela 2 apresenta as dez principais áreas temáticas de acordo com o número de publicações inseridas nelas durante o período de estudo. As princi- pais áreas temáticas de publicação são: Engenharia Civil, Ciência dos Materiais Multidisciplinar e Tecnologia da Construção. Algumas publicações foram classificadas em mais de uma área temática; por conta disso, o número total de registros excede o total de publicações.

Já no que se refere à forma em que as publicações foram encontradas na WOS, os resultados mostraram as maiores taxas em artigos $(73,91 \%)$ e procedimentos $(25,54 \%)$, sendo o restante divididos em revisões e material editorial.

\section{I.2 Publicações POR ANo}

A Figura 1 mostra o número de documentos publicados por ano de acordo com a pesquisa na WOS. A partir da sua análise, percebe-se que o maior número de publicações aconteceu no ano de 2018. Isso mostra que o assunto tem interesse crescente, sendo de relevância no período atual.

\section{I.3 PRINCIPAIS AUtORES}

Excluindo-se as publicações anônimas, os autores que publicaram a maioria dos documentos sobre o processo de corrosão em estruturas de concreto

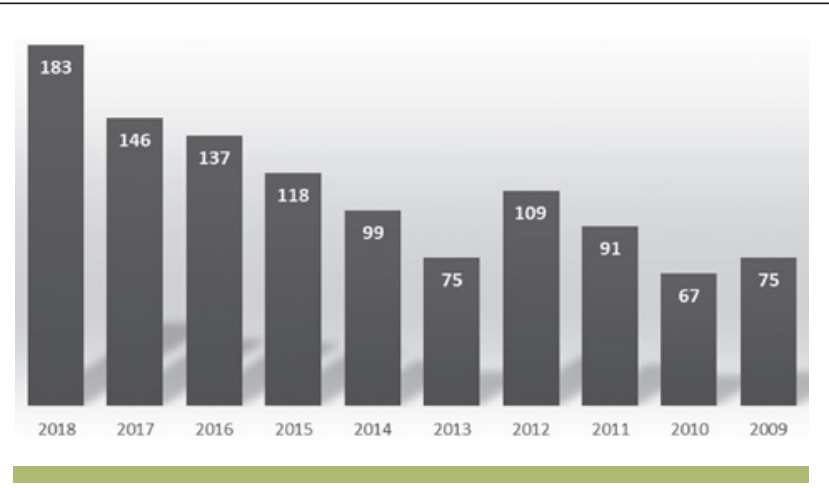

Figura 1

Publicações por ano

Fonte: Web of Science (2019) armado provoca-

Raoul Francis apresentou 24 publicações no período analisado, sendo o autor que possui o maior número de publicações. O autor possui graduação em Engenharia Civil pela École Normale Supérieure de Cachan (1979-1982) e doutorado em Engenharia Civil pela Université Paul Sabatier (1983-1987). É professor há 22 anos no Institut National de Sciences Appliquées (INSA), onde foi diretor de pesquisa do período de 2007 a 2017 e diretor do departamento de Engenharia Civil de 1999 a 2007.

\section{I.4 Título dAs fontes de PUBLICAÇ̃̃es}

A Tabela 4 apresenta as principais fontes de publicações relacionadas à temática analisada.

No banco de dados da WOS, as publicações são encontradas principalmente nos seguintes periódicos: Construction and Building Materials, Materials and Corrosion, Structure and Infrastructure Engineering e Cement and Concrete Composites. A revista Construction and Building Materials se destaca concentrando $12 \%$ das

Tabela 3 - Número de artigos publicados por autor da pela ação de cloretos, nestes últimos dez anos, são apresentados na Tabela 3. Dos 25 principais autores, destaca-se Raoul François, Dan M. Frangopol, Mohammad Shekarchi e Luca Bertolini.

\begin{tabular}{|c|c|}
\hline \multicolumn{2}{|c|}{$\begin{array}{l}\text { Tabela } 3 \text { - Número de artigos } \\
\text { publicados por autor }\end{array}$} \\
\hline Autores & Registros \\
\hline Francois $\mathrm{R}$ & 24 \\
\hline Frangopol DM & 18 \\
\hline Shekarchi M & 18 \\
\hline Bertolini L & 17 \\
\hline Hussain RR & 16 \\
\hline Bastidas - Arteaga E & 15 \\
\hline Jin WL & 15 \\
\hline Stewart MG & 14 \\
\hline Kwon SJ & 12 \\
\hline Zhao TJ & 12 \\
\hline Fonte: Web of Science (2019) & \\
\hline
\end{tabular}




\begin{tabular}{|l|l|}
\hline $\begin{array}{l}\text { Tabela } 4 \text { - Principais fontes } \\
\text { de publicação }\end{array}$ \\
\hline $\begin{array}{c}\text { Áreas de pesquisa } \\
\text { Construction and Building } \\
\text { Materials }\end{array}$ & 132 \\
\hline $\begin{array}{c}\text { Materials and Corrosion } \\
\text { Structure and Infrastructure } \\
\text { Engineering }\end{array}$ & 34 \\
\hline $\begin{array}{c}\text { Cement Concrete Composites } \\
\text { International Journal of }\end{array}$ & 33 \\
\hline Electrochemical Science & 30 \\
\hline Engineering Structures & 27 \\
\hline Journal of Materials in Civil & 26 \\
Engineering & 25 \\
\hline Corrosion Science & 25 \\
\hline Materials and Structures & 19 \\
\hline Advanced Materials Research & \\
\hline Fonte: Web of Science (2019) & \\
\hline
\end{tabular}

1100 publicações encontradas. Tal periódico é reconhecido internacionalmente e possui o intuito de divulgar conhecimento científico na investigação e

Tabela 5 - Principais instituições onde os autores estão associados

\begin{tabular}{|lc|}
\hline Áreas de pesquisa & Registros \\
\hline $\begin{array}{c}\text { Polytechnic University of Milan } \\
\text { Centre National de la }\end{array}$ & 37 \\
Recherche Scientifique CNRS & 28 \\
\hline Université de Toulouse & 26 \\
\hline Zhejiang University & 26 \\
\hline $\begin{array}{c}\text { Institut National des Sciences } \\
\text { Appliquées de Toulouse }\end{array}$ & 24 \\
\hline $\begin{array}{c}\text { Université Federale Toulouse } \\
\text { Midi Pyrenées Comue }\end{array}$ & 24 \\
\hline $\begin{array}{c}\text { Université Toulouse III } \\
\text { Paul Sabatier }\end{array}$ & 24 \\
\hline $\begin{array}{c}\text { Council of Scientific Industrial } \\
\text { Research CSIR India }\end{array}$ & 23 \\
\hline Indian Institute of Technology \\
System IIT System
\end{tabular}

uso de materiais inovadores e de reparo na construção civil.

\section{I.5 PrincipaIS INSTITUIÇõES COM PUBLICAÇ̃̃o NA TEMÁTICA}

A Tabela 5 apresenta as instituições nas quais os autores que publicaram estão associados.

As instituições que mais se destacaram no WOS são: Polytechnic University of Milan (Itália), Centre National de La Recherche Scientifique CNRS (França), Université de Toulouse (França) e Zhejiang University (China).

A Instituição brasileira que mais se destacou no número de publicações no período analisado é a Universidade de São Paulo (USP), com 7 registros, ocupando o $71^{\circ}$ lugar.

\section{I.6 Países de PUBLICACÃo}

Em relação aos países de onde as publicações se originam, a China lidera o ranking, seguida por Estados Unidos, França e Itália, conforme mostra a Tabela 6.

O Brasil ocupa a 19a posição, com

\begin{tabular}{|c|c|}
\hline \multicolumn{2}{|c|}{$\begin{array}{l}\text { Tabela } 6 \text { - Classificação dos } \\
\text { países em relação à quantidade } \\
\text { de publicações }\end{array}$} \\
\hline Países & Registros \\
\hline China & 260 \\
\hline EUA & 141 \\
\hline França & 74 \\
\hline Itália & 61 \\
\hline Índia & 53 \\
\hline Alemanha & 52 \\
\hline Coréia do Sul & 50 \\
\hline Espanha & 50 \\
\hline Inglaterra & 48 \\
\hline Austrália & 43 \\
\hline Fonte: Web of Science (2019) & \\
\hline
\end{tabular}

19 publicações registradas na WOS. Além disso, destaca-se que os países elencados pertencem a diferentes regiões do mundo e vários continentes, o que demonstra a importância dada ao assunto em diferentes locais.

\subsection{Os "Hot Topics"}

Nesta etapa, foram investigados os principais tópicos relacionados à

\section{> Tabela 7 - Análise dos "Hot Topics" nas áreas temáticas}

\begin{tabular}{|cccc|}
\hline Áreas de pesquisa & Número publicações & hb & m \\
\hline Engenharia & 790 & 40 & 4,00 \\
\hline Ciência dos Materiais & 585 & 38 & 3,80 \\
\hline Tecnologia da Construção & 455 & 35 & 3,50 \\
\hline Metalurgia e Engenharia Metalúrgica & 106 & 22 & 2,20 \\
\hline Eletroquímica & 54 & 12 & 1,20 \\
\hline Química & 29 & 7 & 0,70 \\
\hline Ciência Tecnologia e Outros Tópicos & 24 & 7 & 0,70 \\
\hline Mecânica & 23 & 4 & 0,40 \\
\hline Ciência da Computação & 21 & 7 & 0,70 \\
\hline Física & 18 & 5 & 0,50 \\
\hline Fonte: Web of Science (2019) & & & \\
\hline
\end{tabular}


corrosão de armaduras em estruturas de concreto armado por meio da ação de íons cloro, publicados nos últimos anos em relação às suas categorias de estudo. Com base em uma análise preliminar na WOS, foram selecionadas
Tabela 8 - Publicações mais citadas no período analisado

\begin{tabular}{|c|c|c|}
\hline$n^{\circ}$ & Título / Veículo / DOI / Autor & $\begin{array}{l}\text { Número de } \\
\text { citações }\end{array}$ \\
\hline 1 &  & 119 \\
\hline 2 & $\begin{array}{c}\text { Surface treatment of reinforced concrete in marine environment: Influence } \\
\text { on chloride diffusion coefficient and capillary water absorption } \\
\text { In: Construction and Building Materials. } 2009 \\
\text { DOI: https://doi.org/10.1016/j.conbuildmat.2008.06.013 } \\
\text { Por: Medeiros, M. H. F. e Helene, P. }\end{array}$ & 111 \\
\hline 3 & $\begin{array}{l}\text { Service life prediction of concrete wharves with early-aged crack: Probabi- } \\
\text { listic approach for chloride diffusion } \\
\text { In: Structural Safety. } 2009 \\
\text { DOI: https://doi.org/10.1016/j.strusafe.2008.03.004 } \\
\text { Por: Kwon, S. J., Na, U. J., Park, S. S. e Jung, S. H. }\end{array}$ & 106 \\
\hline 4 & $\begin{array}{l}\text { The electrochemical behaviour of stainless steel AISI } 304 \text { in alkaline solu- } \\
\text { tions with different pH in the presence of chlorides } \\
\text { In: Electrochimica Acta. } 2011 \\
\text { DOI: https://doi.org/10.1016/j.electacta.2011.02.094 } \\
\text { Por: Freire, L., Carmezim, M. J., Ferreira, M. G. S. e Montemor, M. F. }\end{array}$ & 104 \\
\hline 5 & $\begin{array}{l}\text { Climate change impact and risks of concrete infrastructure deterioration } \\
\text { In: Engineering Structures. } 2011 \\
\text { D0I: https://doi.org/10.1016/j.engstruct.2011.01.010 } \\
\text { Por: Stewart, M. G., Wang, X. e Nguyen, M. N. }\end{array}$ & 98 \\
\hline 6 & $\begin{array}{l}\text { The importance of chloride content at the concrete surface in assessing the } \\
\text { time to corrosion of steel in concrete structures } \\
\text { In: Construction and Building Materials. } 2009 \\
\text { D0I: https://doi.org/10.1016/j.conbuildmat.2007.12.014 } \\
\text { Por: Ann, K. Y., Ahn, J. H. e Ryou, J. S. }\end{array}$ & 97 \\
\hline
\end{tabular}

Experimental and numerical determination of the chloride penetration in cracked concrete

7 In: Construction and Building Materials. 2009

DOI: https://doi.org/10.1016/j.conbuildmat.2007.12.015

Por: Marsavina, L., Audenaert, K., De Schutter, G., Faur, N. e Marsavina, D.

Seismic fragility estimates for reinforced concrete bridges subject to corrosion

8 In: Structural Safety. 2009

DOl: https://doi.org/10.1016/j.strusafe.2008.10.001

Por: Choe, D., Gardoni, P., Rosowsky, D. e Haukaas, T.

Probabilistic lifetime assessment of RC structures under coupled corrosion-fatigue deterioration processes In: Structural Safety. 2009

DOl: https://doi.org/10.1016/i.strusafe.2008.04.001

Por: Bastidas-Arteaga, E., Bressolette, P., Chateauneuf, A. e Sanchez-Silva, M.

Prediction of time dependent chloride transport in concrete structures exposed to a marine environment

10

DOI: https://doi.org/10.1016/j.cemconres.2009.09.023

Por: Pack, S., Jung, M., Song, H., Kim, S. e Ann, K. Y.

Fonte: Web of Science (2019)

10 categorias de estudo, as quais foram combinadas com o operador booleano de pesquisa. A Tabela 7 apresenta o número de publicações nas categorias de estudo e seus índices $h b$ e $m$.

Baseando-se nas orientações de Banks (2006), é possível classificar como "Hot Topics" as seguintes áreas de engenharia: engenharia, ciência dos materiais, tecnologia da construção e engenharia metalúrgica. Já as áreas de eletroquímica, química, outros tópicos de ciência e tecnologia, mecânica e ciência da computação apresentaram um fator $0,5<m \leq 2$; assim, provavelmente podem se tornar um "Hot Topic" como área de pesquisa na qual a comunidade atuante é grande ou a combinação apresenta recursos muito interessantes. Já a área temática da física apresentou um índice $m$ entre 0 e 0,5, ou seja, trata-se de um tema que pode ser de interesse dos pesquisadores atuantes em um campo específico, que inclui uma pequena comunidade acadêmica.

\subsection{Análise das publicações mais citadas}

Nesta etapa foram selecionados, de acordo com a base de dados consultada, as dez publicações mais citadas, no período analisado, conforme Tabela 8.

Esse levantamento, primeiramente, teve o intuito de verificar a relação entre os trabalhos com maior número de citações e os autores que mais publicaram no período, conforme apresentado anteriormente na Tabela 3. Constatou-se que dos 25 autores com maior número de publicações, 5 estão entre os mais 
citados, sendo eles: Ormellese, Marco, Brenna, Andrea e Kwon, Seung Jun, Bastidas-Arteaga, Emilio e Ann, Ki Yong. Este último é autor de dois artigos que estão entre os mais citados. Além disso, destaca-se que a autoria da segunda publicação mais citada é brasileira, sendo o artigo elaborado por Marcelo Medeiros e Paulo Helene.

No que diz respeito ao conteúdo das cinco publicações mais citadas, os trabalhos desenvolvidos por Ormellese et. al. (2009) e Medeiros e Helene (2009) envolvem estudos que visam inibir a penetração de cloretos. Já o publicado por Kwon et. al. (2009) objetiva, a partir de modelos probabilísticos, prever a vida útil de estruturas inseridas em ambientes com presença de cloretos. O estudo realizado por Stewart, Wang e Nguyen (2011) apresenta a influência das mudanças climáticas no aumento do fenômeno de corrosão de armaduras por ação de cloretos e carbonatação. Por fim, Freire et. al. (2011) analisam o comportamento de vergalhões em aço inoxidável expostos a ação de cloretos com diferentes $\mathrm{pH}$.

\section{CONSIDERAÇÕES FINAIS}

Este estudo ajudou a confirmar que a temática de corrosão de armaduras em estruturas de concreto armado por ação de cloretos está evoluindo, haja vista o crescente número de publicações desse tópico de 2014 a 2018. A China lidera o ranking dos países que mais publicaram sobre o referido tema. Contudo, Estados Unidos, França e Itália também possuem publicações relevantes na área. São considerados "Hot Topics" - tópicos que atingem não apenas suas próprias áreas de pesquisa, mas outras áreas e provavelmente possuem efeitos de aplicação ou características únicas - as áreas de engenharia, ciência dos materiais, tecnologia da construção e engenharia metalúrgica. Já as áreas de eletroquímica, química, outros tópicos de ciência e tecnologia, mecânica e ciência da computação estão na eminência para se tornar um possível "Hot Topics", ou seja, apresentam uma comunidade atuante muito grande ou a combinação da área com a temática apresenta recursos muito interessantes. Dessa forma, percebe-se um interesse na temática que vai além da área de Engenharia Civil. Em relação as publicações mais citadas, foi possível perceber que dos vinte e cinco autores que mais publicaram cinco estão entre os mais citados. No que diz respeito ao conteúdo das publicações mais citadas no período analisado, destacam-se estudos de inibidores de corrosão, modelos probabilísticos para prever a vida útil das estruturas, parâmetros que influenciam na penetração de cloretos e desempenho de materiais especiais inseridos em ambientes em condições especiais com presença de cloretos. Além disso, durante o estudo foi possível verificar a utilidade de mecanismos de pesquisa como a WOS, a qual é uma ferramenta de realização de pesquisas e acesso a publicações para a comunidade acadêmica, bem como auxilia na busca de informações sobre a evolução de temas de interesse. Quanto as limitações da pesquisa, destaca-se a utilização de somente um banco de dados; por este motivo, sugere-se para trabalhos futuros a utilização de outros bancos de dados científicos.

\section{DREFERÊNCIAS BIBLIOGRÁFICAS}

[1] ARAÚJO, C. A. A. Bibliometria: evolução histórica e questões atuais. Em questão, v. 12, n. 1, p. 11-32, 2006.

[2] BANKS, M. G. An extension of the Hirsch index: Indexing scientific topics and compounds. Scientometrics, v. 69, n. 1, p. 161-168, 2006.

[3] FREIRE, L.; CARMEZIM, M. J.; FERREIRA, M. G.; MONTEMOR, M. F. The electrochemical behaviour of stainless steel AISI 304 in alkaline solutions with different pH in the presence of chlorides. Electrochimica Acta, v. 56, n. 14, p. 5280-5289, 2011

[4] HIRSCH, J. E. An index to quantify an individual's scientific research output. Proceedings of the National academy of Sciences, v. 102, n. 46, p. 16569-16572, 2005.

[5] KWON, S. J.; NA, U. J.; PARK, S. S.; JUNG, S. H. et al. Service life prediction of concrete wharves with early-aged crack: Probabilistic approach for chloride diffusion Structural Safety, v. 31, n. 1, p. 75-83, 2009.

[6] MEDEIROS, M.H.F.; HELENE, P. Surface treatment of reinforced concrete in marine environment: Influence on chloride diffusion coefficient and capillary water absorption. Construction and building materials, v. 23, n. 3, p. 1476-1484, 2009.

[7] NEPOMUCENO, A. A. Mecanismos de transporte de fluídos no concreto In: Concreto: Ensino, Pesquisa e Realizações. 1.ed. São Paulo: IBRACON, 2005 cap 26, p. 793-827.

[8] ORMELLESE, M.; LAZZARI, L.; GOIDANICH, S.; FUMAGALLI, G.; BRENNA, A. A study of organic substances as inhibitors for chloride-induced corrosion in concrete. Corrosion Science, v. 51, n. 12, p. 2959-2968, 2009.

[9] RIBEIRO, D. V.; CASCUDO, 0. Durabilidade e vida útil das estruturas de concreto. In: Corrosão e degradação em estruturas de concreto: teoria, controle, técnicas de análise e intervenção. 2.ed. Rio de Janeiro: Elsevier, 2018. cap 03, p. 33-50.

[10] STEWART, M. G.; WANG, X.; NGUYEN, M. N. Climate change impact and risks of concrete infrastructure deterioration. Engineering Structures, v. 33, n. 4, p. 1326-1337, 2011. 